\title{
La región intergénica del gen $H 2 A$ apoya las subpoblaciones KP1(-) y KP1(+) de Trypanosoma rangeli
}

\author{
Brian Alejandro Suárez, Claudia Liliana Cuervo, Concepción Judith Puerta \\ Laboratorio de Parasitología Molecular, Departamento de Microbiología, Facultad de Ciencias, Pontificia \\ Universidad Javeriana, Bogotá, D. C., Colombia \\ Introducción. Con base en la amplificación del ADN de los minicírculos del cinetoplasto y de \\ los genes miniexón, Trypanosoma rangeli ha sido clasificado en las subpoblaciones KP1(-) y \\ $\mathrm{KP} 1(+)$. \\ Objetivo. Comparar la región intergénica de los genes $H 2 A$ entre cepas KP1(+) y KP1(-) de $T$. \\ rangeli, con el fin de aportar evidencias a dicha división. \\ Materiales y métodos. Se amplificó, clonó y determinó la secuencia de la región intergénica \\ de los genes h2a de las cepas KP1(-) Tre y 5048 y de la cepa Choachí [KP1(+)]. Dichas \\ secuencias, junto con las reportadas para las cepas $\mathrm{C} 23$ [KP1(-)] y H14 [KP1(+)], fueron utilizadas \\ para la reconstrucción de árboles filogenéticos basados en los métodos de neighbor-joining, \\ máxima parsimonia y máxima verosimilitud, utilizando la cepa $Y$ de Trypanosoma cruzi como \\ grupo raíz externo. \\ Resultados. Se evidenció heterogeneidad intraespecífica en el tamaño de la región estudiada, \\ soportados por valores bootstrap de $85 \%$ (neighbor-joining), $66 \%$ (máxima parsimonia) y $57 \%$ \\ (máxima verosimilitud), los resultados indican que las cepas KP1(-) se agrupan aparte, \\ claramente diferenciadas de las cepas KP1(+), las cuales presentan una mayor heterogeneidad \\ intraespecífica en tamaño y secuencia. Además, se encontró mayor proximidad filogenética \\ entre $T$. rangeli y $T$. cruzi que entre $T$. rangeli y Trypanosoma brucei. \\ Conclusiones. Los análisis filogenéticos basados en la región intergénica de los genes h2a de \\ las cepas estudiadas apoyan la división de $T$. rangeli en las subpoblaciones KP1(-) y KP1(+). \\ Sin embargo, se requiere estudiar un mayor número de cepas para confirmar estos hallazgos.
}

Palabras clave: Trypanosoma/genética, ADN intergénico, genes, histonas/genética, secuencia conservada, filogenia.

\section{The intergenic region of the histone h2a gene supports two major lineages of Trypanosoma rangeli}

Introduction. Trypanosoma rangeli has been classified in the KP1(+) and KP1(-) subpopulations, based on the mini-exon gene and kinetoplast DNA minicircle amplification profiles.

Objective. The intergenic region of the histone h2a gene was compared between KP1(+) and $\mathrm{KP} 1(-)$ strains of $T$. rangeli to substantiate this classification.

Materials and methods. The amplification, cloning and sequencing of the h2a gene intergenic region was undertaken for the Tre and 5048 [KP1(-)] strains for comparison with the Choachí [KP1(+)] strain. These sequences, along with those previously reported for the KP1 (+) and KP1 (-) $\mathrm{H} 14$ and $\mathrm{C} 23$ strains, were used to reconstruct phylogenetic trees based on the "neighborjoining", maximum parsimony and maximum likelihood methods. The Y strain of Trypanosoma cruzi was chosen as the outgroup.

Results. Intra-specific heterogeneity was observed in the size of the gene region under study, supported by bootstarp values of $85 \%$ (neighbor-joining), $66 \%$ (maximum parsimony) and $57 \%$ (maximum likelihood). The KP1(-) strains were grouped apart, clearly differentiated from the KP1(+) strains. The latter demonstrated a higher intra-specific heterogeneity, both in sequence length and composition. In addition, a closer phylogenetic relationship between $T$. rangeli and $T$. cruzi was found to be more closely related to one another than to $T$. rangeli and Trypanosoma brucei. 
Conclusion. Phylogenetic analyses of analyzed strains based on the intergenic region of the $h 2 a$ genes supported the $T$. rangeli grouping in two major subpopulations known as KP1(+) and KP1(-) strains. However, a higher number of strains are needed to confirm this finding.

Key words: Trypanosoma/genetics, DNA, intergenic, genes, histones/genetics, conserved sequence, phylogeny.

Trypanosoma rangeli es un protozoario hemoflagelado que infecta al hombre, los animales silvestres, los domésticos y los insectos vectores en distintas regiones del centro y sur de América (1). Hasta el momento, T. rangeli es considerado como un parásito no patógeno para el humano, contrario a lo que sucede con Trypanosoma cruzi, el agente causal de la enfermedad de Chagas (2). No obstante, $T$. rangeli, además de distribuirse en regiones geográficas endémicas para la enfermedad de Chagas, comparte vectores y reservorios con $T$. cruzi, presentándose infecciones mixtas tanto en el hospedero vertebrado como en el invertebrado $(1,2)$. Lo anterior, sumado a la presencia de reacciones serológicas cruzadas entre ambos tripanosomas (3), dificultan el diagnóstico específico de la infección chagásica (1). Por lo tanto, resulta de gran interés el estudio de las características genotípicas de $T$. rangelicon el fin de dilucidar el panorama evolutivo, filogenético y biológico de dicho parásito.

De esta forma, utilizando herramientas bioquímicas y moleculares como el estudio de las isoenzimas del parásito (4), la amplificación aleatoria de fragmentos de ADN polimórfico (4) y la amplificación del gen miniexón (5), se encontró que las cepas procedentes de Centroamérica y del norte de Suramérica, presentaban variabilidad con respecto a las cepas oriundas de Brasil. Estudios posteriores demostraron, mediante la caracterización de 37 cepas de $T$. rangeli procedentes de Honduras, Panamá, Colombia, Venezuela y Brasil, que éstas se clasifican independientemente de su origen geográfico, en

\footnotetext{
Correspondencia:

Concepción Judith Puerta Bula, Carrera $7^{a}$ No. 43-82, edificio 52, oficina 608, Departamento de Microbiología, Facultad de Ciencias, Pontificia Universidad Javeriana.

Teléfono: (+571) 320 8320, extensión, 4024; fax: (+571) 320 8320, extensión 4021

cpuerta@javeriana.edu.co
}

Recibido: 05/03/07; aceptado: 29/05/07 dos grupos molecularmente definidos como grupo 1, o cepas KP1(-), y grupo 2, o cepas KP1(+), según la presencia o ausencia del minicírculo KP1 en el ADN del cinetoplasto (6).

Mas adelante, Vallejo et al, (7) y Urrea et al. (8), utilizando como marcadores el ADN del cinetoplasto y el gen miniexón, evidenciaron en sus estudios la asociación de los dos grupos 1 y 2 de $T$. rangelicon las diferentes líneas evolutivas de vectores del género Rhodnius. Es así como las cepas KP1(+) se asocian al grupo Prolixus $(R$. prolixus, $R$. neglectus, $R$. nasutus, $R$. domesticus y $R$. robustos), mientras que las cepas KP1(-) se asocian con el grupo Pallescens (R. pallescens, R. ecuadoriensis y R.colombiensis). Datos que están de acuerdo con la teoría de Schofield y Dujardin (9), según la cual las especies de Rhodnius sufrieron un proceso de radiación en Suramérica a partir de especies ancestrales semejantes a $R$. pictipes en la región del Amazonas, de la cual se originaron tres líneas adaptativas del vector representadas por las formas forestales ( $R$. pictipes, $R$. stali, R.brethesi, $R$. paraensis y $R$. dalessandroi), el grupo Prolixus, en el cual se incluyen los vectores que se distribuyen al oriente de la región Andina y el complejo Pallescens, conformado por vectores distribuidos a lo largo de la Cordillera de los Andes. Además, los estudios de la amplificación aleatoria de fragmentos de ADN polimórfico de cepas aisladas de $R$. prolixus, $R$. pallescens, $R$. colombiensis y $R$. ecuadoriensis, muestran que estas cepas se agrupan no sólo de acuerdo con la presencia del minicírculo KP1, sino según la especie de Rhodnius de la cual proceden (10). Se plantea, además, que el vector actúa como un filtro biológico de las subpoblaciones del parásito (11).

Teniendo en cuenta que ensayos previos de reacción en cadena de la polimerasa de baja astringencia usando un único iniciador (LSSPPCR) sugieren que la región intergénica del gen 
H2A puede constituir un marcador diferencial de cepas KP1(-) y KP1(+) del parásito (12), en el presente trabajo se utilizó dicha secuencia para aportar evidencias moleculares basadas en un gen nuclear que soporten la división de $T$. rangeli en las subpoblaciones KP1(-) y KP1(+).

\section{Materiales y métodos}

Amplificación, clonación y determinación de
la secuencia de nucleótidos de la región
intergénica de los genes h2a

Para la amplificación de los fragmentos de interés se utilizaron los ADN genómicos disponibles en la genoteca del Laboratorio de Parasitología Molecular de la Pontificia Universidad Javeriana, correspondientes a las cepas KP1(-): Tre [Morales et al., 2002 (13)] y 5048 [MHOM/CO/99/5048, Morales et al., 2002 (13)] y la cepa Choachí [IRHO/ CO/82/Ch, Schottelius, 1987(14)] KP1(+) de T. rangeli. Los iniciadores empleados, $\mathrm{H} 14$ IntF (5'TAGATACACCTGGGGGAACG 3') y H14 IntR (5'TCACAGCTGCAACAAGCAAAC 3'-) (12), amplifican la región intergénica del gen $H 2 A$ de la cepa H14 comprendida entre los nucleótidos 451 a 764 (No. de acceso al GenBank AY147905).

Para la reacción de amplificación se utilizaron 125 ng de ADN genómico del parásito, 20 pmoles de cada uno de los iniciadores, $2,5 \mu \mathrm{l}$ de solución tampón de reacción $10 \times(10 \mathrm{mM}$ Tris- $\mathrm{HCl}$ pH 9,0 $50 \mathrm{mM} \mathrm{KCl}$, y $0,1 \%$ de tritón X-100), 2,6 U de la enzima High Fidelity PCR System (Roche), 1,5 $\mathrm{mM} \mathrm{MgCl}, 0,2 \mathrm{mM}$ de cada dioxinucleótido trifosfatado (dNTPs), en un volumen final de reacción de $25 \mu$ l. Las reacciones se llevaron a cabo en un termociclador MJ Research PTC-100, usando el siguiente perfil de amplificación: desnaturación inicial a $95^{\circ} \mathrm{C} / 30 \mathrm{~s}$, seguida de 35 ciclos $95^{\circ} \mathrm{C} / 30 \mathrm{~s}, 65^{\circ} \mathrm{C} / 40 \mathrm{~s}$ y $72^{\circ} \mathrm{C} / 40 \mathrm{~s}$, con extensión final a $72^{\circ} \mathrm{C}$ por $5 \mathrm{~min}$.

El producto de amplificación se visualizó mediante electroforesis horizontal en geles de agarosa al $2 \%$, teñidos con bromuro de etidio (15). Los fragmentos de amplificación de interés fueron escindidos y purificados del gel usando el estuche comercial QIA-Gel extraction kit (QIAGEN), para su posterior clonación en el plásmido vector pGEM®-T Easy (Promega) (15). Los clones seleccionados con base en la prueba de blancas y azules (15), uno por cepa, fueron sometidos a extracción del ADN plasmídico total mediante el estuche comercial Wizard® Plus SV Minipreps DNA purification system (Promega); se determinaron dos veces las secuencias de ambas cadenas del ADN del inserto presente en los mismos mediante procedimientos estándares del servicio suministrado por la compañía Macrogen ${ }^{\circledR}$ (Corea), utilizando los iniciadores universales M13F y M13R.

\section{Alineamiento de secuencias y análisis filogenético}

Para confirmar que las secuencias obtenidas para las cepas Tre, 5048 y Choachí codificaran para la región intergénica de los genes $h 2 a$, éstas fueron comparadas con las reportadas en la base de datos GenBank usando la herramienta BLAST-N (16). Posteriormente, dichas secuencias, junto con las previamente reportadas para las cepas $\mathrm{C} 23$ (No. de acceso al GenBank (AF169130) y H14 (No. de acceso al GenBank AY147905) de $T$. rangelie $Y$ de $T$. cruzi (No. de acceso al GenBank AF167074), se alinearon usando el programa CLUSTAL-W versión 1.81 (17), ajustándose manualmente con el programa Bioedit Sequence Alignment Editor (18). La reconstrucción de los árboles se realizó mediante los métodos de neighbor-joining (19) y máxima parsimonia utilizando los programas MEGA 3.1 (20) y PAUP 4.0 con valores bootstrap obtenidos a partir de 1.000 pseudorréplicas y el método de máxima verosimilitud usando el programa PAUP 4.0 (21). Para el árbol de neighbor-joining se usó el modelo de sustitución Kimura de 2-parámetros (22), para el de máxima parsimonia, se usó el nivel 1 de la opción close-neighbor-interchange (CNI, por sus siglas en inglés) con una adición aleatoria para árboles de 10 réplicas y el de máxima verosimilitud fue realizado mediante búsqueda heurística con valores bootstrap obtenidos a partir de 1.000 pseudorréplicas, siguiendo el modelo de sustitución HKY85 de Hasegawa et al. (23).

\section{Resultados}

Amplificación, clonación y determinación de la secuencia de nucleótidos de la región intergénica de los genes h2a

Para cada una de las tres cepas en estudio, se obtuvo el fragmento de amplificación del tamaño 
esperado de alrededor $310 \mathrm{pb}$ (no se muestran los datos), fragmentos que fueron clonados, obteniéndose tamaños de 309, 307 y 324 pb para los insertos de los clones Trec1, c5048-3 y Cho1, derivados de las cepas C23, 5048 y Choachí, respectivamente.

Los análisis BLAST/N de las secuencias obtenidas mostraron porcentajes de similitud de $92 \%$ a 99,5\% (valor e: 2 e- 147 a $3 e-50$ ) con lo reportado para las cepas $\mathrm{C} 23$ y $\mathrm{H} 14$ de $\mathrm{T}$. rangeli, y $55,2 \%$ (valor e: 3 e-05) para la cepa Y de T. cruzi, con lo cual se confirmó que la región intergénica de los genes H2A había sido amplificada y clonada.

\section{Características de la región intergénica de los genes h2a}

En primer lugar, esta región se caracteriza por la existencia de motivos conservados tales como la secuencia potencialmente formadora de la horquilla 3' en el ARN mensajero, previamente descrita para los genes de las histonas (figura 1, nt 12-30), la cual se encuentra implicada en la regulación de la síntesis de las histonas de eucariotes (24), el tracto de polipirimidinas (figura 1, nt 246-269) y 3 sitios "AG" posibles aceptores de la secuencia líder o mini-exon (figura 1, nt 305$306,312-313$ y 322-323), señales que en conjunto han sido descritas en tripanosomátidos y participan en el proceso de trans-splicing, evento requerido por estos parásitos para convertir los ARN mensajeros policistrónicos en monocistrones, antes de su traducción (25).

En segundo término, se observó variabilidad de la secuencia entre las cepas, especialmente a partir de la posición 150, donde en los nucleótidos 150-154 de las cepas KP1(+) mantienen conservada una región de 4 nucleótidos (CTTG), la cual está ausente en las cepas KP1(-); en la posición 264-269, las cepas KP1(+) extienden el tracto de polipirimidinas con seis timinas para la cepa Choachí y cinco para la cepa H14. Por último, en las posiciones 287-288, las cepas KP1 (-) mantienen conservado el dímero GT, el cual se encuentra ausente en las cepas KP1 (+) (figura 1).

También se observaron mutaciones a nivel individual en cada cepa, independientemente de la subpoblación del parásito; la mayoría de ellas fueron cambios en un único nucleótido (SNP, por sus siglas en inglés), concretamente siete transiciones y cuatro eventos de inserción o deleción. En concreto, entre las cepas KP1(-), la cepa C23 presentó una transición de adenina por guanina en la posición 8 , la cepa Tre de citosina por timina en la posición 141 y la cepa 5048 de timina por citosina en la posición 119. Además, esta última cepa presentó dos eventos de inserción/deleción de una timina en las posiciones 163 y 263. Por su parte, en cuanto a las cepas KP1(+), la cepa H14 presentó una transición en la posición 36 de adenina por guanina, de timina por citosina en el nucleótido 260 y de guanina por adenina en la posición 283. La cepa Choachí presentó una transición de guanina por adenina en la posición 98 , un evento de deleción/inserción de una timina en la posición 269 y de siete guaninas en la posición 166-172 (figura 1).

\section{Análisis filogenético}

La relación genética entre las cepas se determinó, en primer lugar, mediante el método de distancia neighbor-joining y se encontró que las cepas $\mathrm{KP} 1(-)$ forman un grupo monofilético distinto de las cepas $\mathrm{KP} 1(+)$, soportado por un valor bootstrap de $85 \%$ (figura $2 \mathrm{~A}$ ); en general, esta topología se mantiene al utilizar los métodos filogenéticos de máxima parsimonia y máxima verosimilitud, en las cuales los valores bootstrap corresponden a $66 \%$ y $57 \%$, respectivamente (figura $2 \mathrm{~B}$ y $2 \mathrm{C}$ ).

Por otra parte, cuando se amplía el número de secuencias de $T$. cruzi por analizar y se utiliza además la secuencia respectiva de Leishmania infantum como grupo raíz externo, se observa, de una parte, que se mantiene la topología del árbol para las cepas KP1(-) y KP1(+) de $T$. rangeli $y$, de otra, que $T$. cruzi y $T$. rangeliforman grupos distintos a partir de un ancestro común, soportados por valores bootstrap de $82 \%$ y $100 \%$, respectivamente (figura 3 ).

\section{Discusión}

Las regiones intergénicas de tripanosomátidos constituyen un blanco interesante de estudio pese a que no tienen funciones codificantes para productos proteicos. Es así como, por ejemplo, se ha reportado que la región 3' no traducida (la 


\begin{tabular}{|c|c|c|c|c|c|}
\hline & & & & & 50 \\
\hline $\mathrm{C} 23$ & tagatacgcc & tgggggaacg & ccattgccca & gccacagtga & ctcacgtgtg \\
\hline 5048 & tagatacacc & tgggggaacg & ccattgccca & gccacagtga & ctcacgtgtg \\
\hline Tre & tagatacacc & tgggggaacg & ccattgccca & gccacagtga & ctcacgtgtg \\
\hline H1 4 & tagatacacc & tgggggaacg & ccattgccca & gccacggtga & ctcacgtgtg \\
\hline \multirow[t]{2}{*}{ Choachí } & tagatacacc & tgggggaacg & ccattgccca & gccacagtga & ctcacgtgtg \\
\hline & & & & & 100 \\
\hline $\mathrm{C} 23$ & gccecataga & atagtgtgtt & tgtttttatt & tatttatttg & tgttggcgtg \\
\hline 5048 & gccecataga & atagtgtgtt & tgtttttatt & tatttatttg & tgttggcgtg \\
\hline Tre & gccccataga & atagtgtgtt & tgtttttatt & tatttatttg & tgttggcgtg \\
\hline H1 4 & gccecataga & atagtgtgtt & tgtttttatt & tatttatttg & tgttggcgtg \\
\hline \multirow[t]{2}{*}{ Choachí } & gccecataga & atagtgtgtt & tgtttttatt & tatttatttg & tgttggcatg \\
\hline & & & & & 150 \\
\hline C23 & gettctccca & atttctcctc & tcgcetctec & cacgggaatg & cgcaacgtc- \\
\hline 5048 & gettctccca & atttctccec & tegcetcte & cacgggaatg & cgcaacgtc- \\
\hline Tre & gettctccca & attetcctc & tagcetcttc & cacgggaatg & tgcaacgtc- \\
\hline H1 4 & gettctccca & atttctcetc & tegcetctec & cacgggaatg & cgcaacgtcc \\
\hline \multirow[t]{2}{*}{ Choachí } & gettctccca & attectcetc & tcgectcttc & cacgggaatg & cgcaacgtcc \\
\hline & & & & & 200 \\
\hline C23 & $---t t t t t t t$ & tttgg ----- & --atttttga & aacattttcg & ccggcacctg \\
\hline 5048 & $---t t t t t t t$ & tt-gg----- & --atttttga & aacattttcg & ccggcacctg \\
\hline Tre & $---t t t t t t$ & tttgg----- & --atttttga & acatttcg & ccggcacctg \\
\hline $\mathrm{H} 14$ & ttgtttttt & tttgg----- & --atttttga & aacattttcg & ccggcacctg \\
\hline \multirow[t]{2}{*}{ Choachí } & ttgtttett & tttggggggg & ggatttttga & aacattteg & ccggcacctg \\
\hline & & & & & 250 \\
\hline C23 & gCGGCGGCGT & GGGTGCTGCG & тстGстTтст & GTGGCTGCAT & GCGTGCTTTC \\
\hline 5048 & gCGGCGGCGT & GCGTGCTGCG & тстGCTTTCT & GTGGCTGCAT & GCGTGCTTTd \\
\hline Tre & gCGGCGGCGT & GCGTGCTGCG & TCTGCTTTCT & GTGGCTGCAT & GCGTGCTTTC \\
\hline $\mathrm{H} 14$ & gCGGCGGCGT & GGGTGCTGCG & TCTGCTTTCT & GTGGCTGCAT & GCGTGCTTTd \\
\hline \multirow[t]{2}{*}{ Choachí } & gCGGCGGCGT & GGGTGCTGCG & TCTGCTTTCT & GTGGCTGCAT & GCGTGCTTTd \\
\hline & & & & & 300 \\
\hline C23 & TССТTTTTTT & TTT------G & тттссттстт & GTGTTGTGTT & TTCTTTGTTC \\
\hline 5048 & тССтTтTтT & $\mathrm{TT}------\mathrm{G}$ & тттССтTстT & GTGTTGTGTT & TTCTTTGTTC \\
\hline Tre & тССтTтTтT & TTT----- $G$ & тттсСтTстT & GTGTTGTGTT & TTCTTTGTTC \\
\hline H1 4 & тсстттттс & Tтттттт-G & тттссттстт & GTATTGT- $-\mathrm{T}$ & TTCTTTGTTT \\
\hline Choachí & тССТTTTTTT & TTTTTTTTTG & $\begin{array}{c}\text { тТТССТТСТT } \\
326\end{array}$ & GTGTTGT--T & TTCTTTGTTT \\
\hline C23 & TTCAAGATCA & CAGCTGCAAC & AAGCAA & & \\
\hline 5048 & TTCAAGATCA & CAGCTGCAAC & AAGCAA & & \\
\hline Tre & TTCAAGATCA & CAGCTGCAAC & AAGCAA & & \\
\hline $\mathrm{H} 14$ & TTCAAGATCA & CAGCTGCAAC & AAGCAA & & \\
\hline Choachí & TTCAAGATCA & $\stackrel{\text { CAGCTGCAAC }}{\bullet}$ & $\stackrel{\text { AAGCAA }}{\bullet}$ & & \\
\hline
\end{tabular}

Figura 1. Múltiple alineamiento de las regiones intergénicas de los genes h2a de las cepas KP1(-) C23, 5048 y Tre y de las cepas KP1(+) H14 y Choachí de Trypanosoma rangeli. Los números a la derecha señalan la posición de cada nucleótido en la secuencia. En letra negrita se denota la secuencia invertida con potencial para formar el stem-loop u horquilla 3', así como también los SNP encontrados. Los dinucleótidos AG aceptores de la secuencia líder se indican mediante el símbolo (•) y el tracto de polipirimidinas se encuentra señalado por un recuadro. En letra minúscula se denota el inicio de la región 3' no codificante y de forma contigua se denota la región 5' no codificante en mayúscula.

cual hace parte de la región intergénica o espaciadora) de los genes codificantes para la calmodulina, acumula mutaciones entre los diferentes linajes de T. cruzi que hacen posible su distinción mediante métodos filogenéticos (26).

La histona $\mathrm{H} 2 \mathrm{~A}$ es una proteína conservada evolutivamente, la cual puede presentar organización genómica diferente según la especie y la cepa (27). En T. cruzi se han encontrado diferencias en el número de copias, organización cromosómica y el nivel de expresión de sus mARN (28). Por su parte, en $T$. rangelise han observado diferencias en la secuencia al comparar la región intergénica del gen $h 2 a$ entre dos cepas (12). Por lo tanto, estas secuencias pueden constituir un marcador nuclear útil para la agrupación y diferenciación de distintas cepas de T. rangeli, además de los actualmente postulados como son el ADNk y el gen mini-exón (5-8).

Dentro del anterior contexto, en este trabajo se estudiaron las relaciones filogenéticas entre cepas $\mathrm{KP} 1(+)$ y KP1(-) de $T$. rangeli usando como base la región intergénica de los genes codificantes para la histona H2A del parásito. Se detectaron 

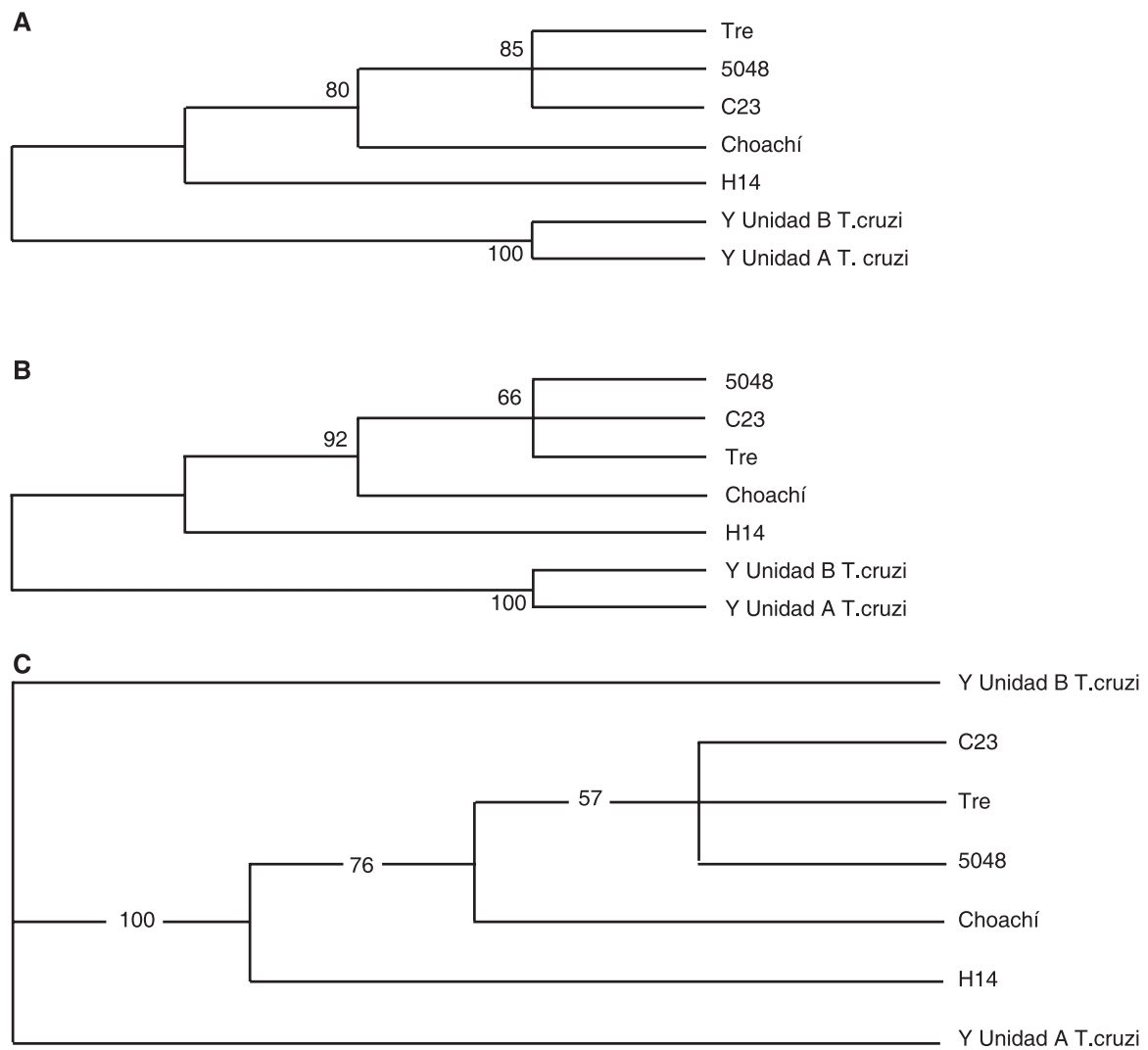

Figura 2. Análisis filogenéticos de $T$. rangeli basados en la secuencia de la región intergénica de los genes $h 2 a$ utilizando las secuencias respectivas de las subunidades codificantes de 0,76 (unidad A) y 1,2 kb (unidad B) para la histona H2A en la cepa Y de T. cruzi como grupo raíz externo (No. de acceso al GenBank AF167074), con valores bootstrap obtenidos a partir de 1.000 pseudorréplicas. (A) Árbol de neighbor-joining utilizando el programa MEGA 3.1 (B) Árbol de máxima parsimonia utilizando el programa MEGA 3.1. (C) Árbol de máxima verosimilitud utilizando el programa PAUP 4.0.

diferencias en el tamaño de los fragmentos amplificados al utilizar los iniciadores $\mathrm{H} 14 \mathrm{IntF} / \mathrm{R}$. Se encontró un mayor tamaño en las cepas KP1(+) Choachí y H14 (12) que en las cepas KP1(-) Tre, 5048 y C23 (29), y estas últimas secuencias mostraron una menor dispersión en sus tamaños. Hubo diferencias caracterizadas principalmente por eventos de inserción/deleción, en los cuales, para el caso de las cepas $\mathrm{KP} 1(+)$, no existe homogeneidad en dicho polimorfismo ya que en la cepa Choachí existe una inserción de siete nucleótidos de guanina en la posición 166 ausente en la cepa H14. Estos resultados sugieren una mayor heterogeneidad dentro del grupo KP1(+), comparado con las cepas KP1(-). Este hecho, aun cuando debe confirmarse utilizando un mayor número de cepas, concuerda con estudios de cariotipo en los cuales, al analizar los cromosomas de ambas subpoblaciones del parásito mediante electroforesis de campo pulsado, las cepas KP1(+) exhiben heterogeneidad entre sus perfiles de migración, mientras que las cepas KP1(-) presentan un patrón electroforético homogéneo (30).

De especial interés es el hecho de que las regiones intergénicas presentaron variabilidad en su secuencia, principalmente a partir del nucleótido 150, manteniendo bastante conservada su región $5^{\prime}$, en la cual es posible que se encuentren, además de la horquilla 3' previamente descrita en eucariotes superiores, señales implicadas en la regulación postranscripcional de 


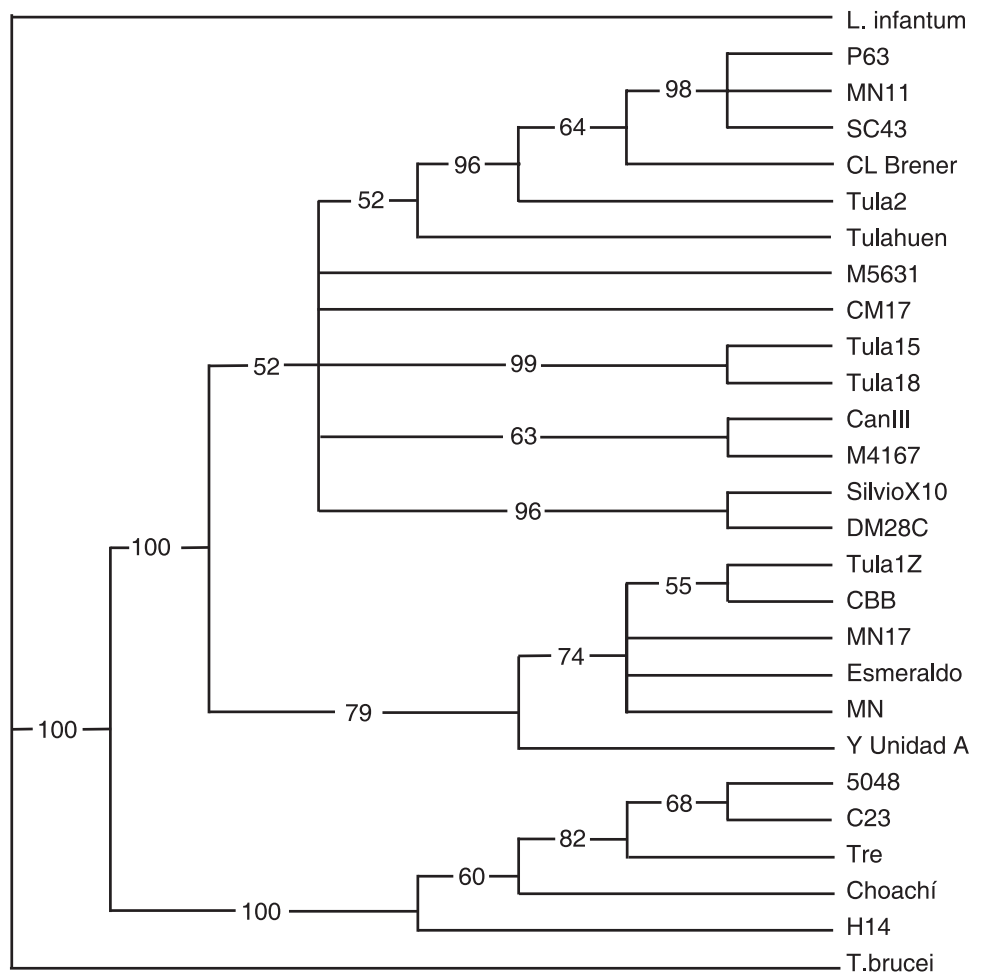

Figura 3. Análisis filogenético de T. rangeli y $T$. cruzi basados en las secuencias intergénicas de los genes $h 2 a$ usando las secuencias respectivas de Leishmania infantum como grupo raíz externo. Árbol de neighbor-joining" con valores bootstrap obtenidos a partir de 1.000 pseudo-réplicas, utilizando el programa PAUP 4.0. T. rangeli: cepa Tre (EF100609), 5048 (EF100610), C23 (AF169130), Choachí (EF600611), H14 (AY147905); T. cruzi: cepa P63 (AY540658), MN (AY540657), MN11 (DQ021891), MN17 (DQ021892), SC43 (AY540659), CL Brener (AF545081), Tulahuen (AY540660), Tula 2 (DQ021887), Tula 12 (DQ021888), Tula 15 (DQ021889), Tula 18 (DQ021890), M5631 (AY540661), Silvio X10 (AF545080), DM28C (AF545079), CM17 (AY540656), Can III (AY540654), M4167 (AY540653), CBB (AY540655), Esmeraldo (AF545082), Y unidad A (AF167074); T. brucei (AY159408) y L. infantum (AJ419627).

estos genes, tal como se ha reportado previamente para otros genes en tripanosomátidos (31).

Estudios filogenéticos mediante el método de distancia neighbor-joining y los discretos de máxima parsimonia y máxima verosimilitud, permiten dilucidar que las cepas KP1(-) estudiadas constituyen un grupo monofilogenético, distinto al de las cepas $\mathrm{KP} 1(+)$, sustentado por altos valores bootstrap. Estos resultados apoyan la existencia de las subpoblaciones KP1(-) y KP1(+) de $T$. rangeli. Sin embargo, es importante resaltar que se requiere aumentar el número de cepas para generalizar estos hallazgos.

Además, al realizar análisis filogenéticos utilizando secuencias reportadas en el GenBank para T. cruzi, Trypanosoma brucei y L. infantum, entre otras, se evidenció cómo las diferencias en la región intergénica del gen codificante para la histona $\mathrm{H} 2 \mathrm{~A}$ también permiten distinguir $T$. rangeli de $T$. cruzi. Este resultado está de acuerdo con la presencia exclusiva del elemento SIRE (short interspersed repetitive element), en la región 3' no traducida de estos genes en T. cruzi (32).

Por otro lado, estudios filogenéticos basados en las secuencias de ADN de la subunidad pequeña ribosomal y del espaciador interno transcrito ITS1, mostraron que el grupo monofilogenético que agrupa las cepas de $T$. rangeli se encuentra más cercano a T. cruzi (subgénero Schyzotrypanum) que a T. brucei (subgénero Trypanozoon) (33). En este sentido, es importante anotar que en este estudio se evidenció una mayor proximidad 
filogenética entre $T$. rangeli y $T$. cruzi que entre $T$. rangeli y $T$. brucei, a pesar de que estos dos últimos parásitos son trasmitidos por la saliva del vector (sección salivaria), mientras que $T$. cruzi se transmite por las heces de los triatóminos (sección stercoraria).

De especial interés es el hecho de que las cepas $\mathrm{KP} 1(+)$ y KP1(-) de $T$. rangeliparecen corresponder a dos de los cuatro grupos descritos para $T$. rangeli por Maia da Silva et al. mediante análisis de la amplificación aleatoria de fragmentos de ADN polimórfico, en concreto a los grupos denominados como A y C, respectivamente (34).

Por lo tanto, se requiere aumentar el número de cepas, incluyendo aislamientos procedentes de diversos orígenes geográficos y hospederos, para confirmar estos hallazgos y dilucidar la posible presencia de otras subpoblaciones del parásito.

\section{Agradecimientos}

Se agradece a Rubén Santiago Nicholls y Marleny Montilla del Laboratorio de Parasitología del Instituto Nacional de Salud por proporcionar las cepas de $\mathrm{T}$. rangeli para la constitución de la genoteca; a Gustavo Adolfo Vallejo del Laboratorio de Investigación en Parasitología Tropical de la Universidad del Tolima por la caracterización de las mismas; al Departamento de Sistemas de la Pontificia Universidad Javeriana por su colaboración con la ejecución de los programas computacionales; y a Diana Álvarez de la Pontificia Universidad Javeriana y Felipe Guhl del Centro de Investigaciones en Microbiología y Parasitología Tropical (CIMPAT) de la Universidad de los Andes, por sus valiosos aportes y comentarios al trabajo.

\section{Conflicto de intereses}

Los autores manifestamos expresamente que durante la realización del presente trabajo no existió conflicto de interés alguno que pudiera afectar los resultados obtenidos.

\section{Financiación}

Este trabajo fue financiado por la Vicerrectoría Académica de la Pontificia Universidad Javeriana, proyecto No. 1038.

\section{Referencias}

1. Guhl F, Vallejo GA. Trypanosoma (Herpetosoma) rangeli Tejera, 1920: an updated review. Mem Inst Oswaldo Cruz. 2003;98:435-42.

2. D'Alessandro-Bacigalupo A, Gore-Saravia N. Trypanosoma rangeli. En: Gilles HM, editor. Protozoal diseases. New York: Arnold Publishers; 1999. p.398-410.

3. Basso B, Moretti ER, Vottero-Cima E. Immune response and Trypanosoma cruzi infection in Trypanosoma rangeli-immunized mice. Am J Trop Med Hyg. 1991;44:413-9.

4. Steindel M, Dias Neto E, Pinto CJ, Grisard EC, Menezes CL, Murta SM, et al. Randomly amplified polymorphic DNA (RAPD) and isoenzyme analysis of Trypanosoma rangeli strains. J Eukaryot Microbiol. 1994;41:261-7.

5. Grisard EC, Campbell DA, Romanha AJ. Mini-exon gene sequence polymorphism among Trypanosoma rangeli strains isolated from distinct geographical regions. Parasitology. 1999;118:375-82.

6. Vallejo GA, GuhI F, Carranza JC, Lozano LE, Sánchez JL, Jaramillo JC, et al. kDNA markers define two major Trypanosoma rangeli lineages in Latin America. Acta Trop. 2002;81:77-82.

7. Vallejo GA, Guhl F, Carranza JC, Moreno J, Triana O, Grisard EC. Parity between kinetoplast DNA and mini-exon gene sequences supports either clonal evolution or speciation in Trypanosoma rangeli strains isolated from Rhodnius colombiensis, $R$. Pallescens and $R$. prolixus in Colombia. Infect Genet Evol. 2003;3:39-45.

8. Urrea DA, Carranza JC, Cuba CA, GurgelGonçalves R, Guhl F, Schofield CJ, et al. Molecular characterisation of Trypanosoma rangeli strains isolated from Rhodnius ecuadoriensis in Perú, $R$. colombiensis in Colombia and R. pallescens in Panamá, supports a co-evolutionary association between parasites and vectors. Infect Genet Evol. 2005;5:123-9.

9. Schofield CJ, Dujardin JP. Theories on the evolution of Rhodnius. Actualizaciones Biológicas. 1999,21:183-97.

10. Vallejo GA, Guhl F, Carranza JC, Triana O, Pérez G, Ortiz PA, et al. Interacción tripanosoma-vectorvertebrado y su relación con la sistemática y la epidemiología de la tripanosomiasis americana. Biomédica. 2007;27 (Suppl. 1):110-8.

11. Sánchez IP, Pulido XC, Carranza JC, Triana O, Vallejo GA. Inmunidad natural de Rhodnius prolixus (Hemiptera: Reduviidae: Triatominae) frente a la infección con Trypanosoma (Herpetosoma) rangeli KP1(-) aislados de Rhodnius pallescens, $R$. colombiensis y $R$. ecuadoriensis. Revista de la Asociación Colombiana de Ciencias Biológicas. 2005;17:108-18.

12. Cuervo C, López MC, Puerta C. The Trypanosoma rangeli histone $\mathrm{H} 2 \mathrm{~A}$ gene sequence serves as a dif- 
ferential marker for KP1 strains. Infect Genet Evol. 2006;6:401-9

13. Morales L, Romero I, Diez H, Del Portillo P, Montilla M, Nicholls S, et al. Characterization of a candidate Trypanosoma rangeli small nucleolar RNA gene and its application in a PCR-based parasite detection. Exp Parasitol. 2002;102:72-80.

14. Schottelius J. Neuraminidase fluorescence test for differentiation of Trypanosoma cruzi and Trypanosoma rangeli. Trop Med Parasitol. 1987;38:323-7.

15. Puerta C, Urueña C. Prácticas de Biología Molecular. Colección Biblioteca del Profesional. Bogotá: Editorial Pontificia Universidad Javeriana; 2005. p.24-54.

16. Altschul SF, Madden TL, Schaffer AA, Zhang J, Zhang Z, Miller W, et al. Gapped BLAST and PSIBLAST: A new generation of protein database search programs. Nucleic Acids Res. 1997;25:3389-402.

17. Thompson JD, Higgins DG, Gibson TJ. CLUSTAL $\mathrm{W}$ : improving the sensitivity of progressive multiple sequence alignment through sequences weighting, position specific gap penalties and weight matrix choice. Nucleic Acids Res. 1994.;22:4673-80.

18. Hall TA. BioEdit: a user-friendly biological sequence alignment editor and analysis program for Windows 95/ 98/NT. Nucleic Acids Symp Ser. 1999;41: 95-8.

19. Saitou $\mathbf{N}, \mathbf{N e i} \mathbf{M}$. The neighbor-joining method: a new method for reconstructing phylogenetic trees. Mol Biol Evol. 1987;4:406-25.

20. Kumar S, Tamura K, Nei M. MEGA3: Integrated software for molecular evolutionary genetics analysis and sequence alignment. Brief Bioinform. 2004; 5:150-63.

21. Felsenstein J. Evolutionary trees from DNA sequences: a maximum likelihood approach. J Mol Evol. 1981;17:368-76.

22. Kimura M. A simple method for estimating evolutionary rates of base substitutions through comparative studies of nucleotide sequences. J Mol Evol. 1980;16:111-20.

23. Hasegawa M, Kishino $\mathbf{H}$, Yano T. Dating of the human-ape splitting by a molecular clock of mitochondrial DNA. J Mol Evol. 1985;22:160-74.

24. Lanzotti DJ, Kupsco JM, Yang XC, Dominski Z, Marzluff WF, Duronio RJ. Drosophila stem-loop binding protein intracellular localization is mediated by phos- phorylation and is required for cell cycle-regulated histone mRNA expression. Mol Biol Cell. 2004;15:1112-23.

25. Agabian N. Trans splicing of nuclear pre-mRNAs. Cell. 1990;61:1157-60.

26. Brandao A, Fernandes O. Trypanosoma cruzi: Mu tations in the $3^{\prime}$ untranslated region of calmodulin gene are specific for lineages $T$. cruzi I, T. cruzi II, and the Zymodeme III isolates. Exp Parasitol. 2006;112:247-52.

27. Maxson R, Cohn R, Kedes L, Mohun T. Expression and organization of histone genes. Annu Rev Genet. 1983;17:239-77

28. Thomas MC, Olivares M, Escalante M, Marañón C, Montilla M, Nicholls S, et al. Plasticity of the histone $\mathrm{H} 2 \mathrm{~A}$ genes in a Brazilian and six Colombian strains of Trypanosoma cruzi. Acta Trop. 2000;75:203-10.

29. Puerta C, Cuervo P, Thomas MC, López MC. Molecular characterization of the Histone $\mathrm{H} 2 \mathrm{~A}$ gene from the parasite Trypanosoma rangeli. Parasitol Res. 2000;86:916-22.

30. Urueña C, Santander SP. Determinación de la localización cromosómica de los genes que codifican para la proteína KMP11 de Trypanosoma rangeli. [Tesis de Pregrado en Bacteriología]. Bogotá: Facultad de Ciencias. Pontificia Universidad Javeriana; 2003.

31. da Silva RA, Bartholomeu DC, Teixeira SM. Control mechanisms of tubulin gene expression in Trypanosoma cruzi. Int J Parasitol. 2006;36:87-96.

32. Vázquez M, Lorenzi H, Schijman AG, Ben-Dov C, Levin MJ. Analysis of the distribution of SIRE in the nuclear genome of Trypanosoma cruzi. Gene. 1999;239:207-16.

33. Da Silva FM, Noyes H, Campaner M, Junqueira AC, Coura JR, Añez N, et al. Phylogeny, taxonomy and grouping of Trypanosoma rangeli isolates from man, triatomines and sylvatic mammals from widespread geographical origin based on SSU and ITS ribosomal sequences. Parasitology. 2004;129:549-61.

34. Maia da Silva F, Rodriguez AC, Campaner M, Takata CS, Brigido MC, Junqueira AC, et al. Randomly amplified polymorphic DNA analysis of Trypanosoma rangeli and allied species from human, monkeys and other sylvatic mammals of the Brazilian Amazon disclosed a new group and a species-specific marker Parasitology. 2004;128:283-94. 